\title{
Real-Time Dynamics of Plasma Balls from Holography
}

\author{
Hans Bantilan®, ${ }^{1,4, *}$ Pau Figueras, ${ }^{1, \dagger}$ and David Mateos ${ }^{2,3, *}$ \\ ${ }^{1}$ School of Mathematical Sciences, Queen Mary University of London, \\ Mile End Road, London E1 4NS, United Kingdom \\ ${ }^{2}$ Departament de Física Quàntica i Astrofísica and Institut de Ciències del Cosmos (ICC), \\ Universitat de Barcelona, Martí i Franquès 1, ES-08028 Barcelona, Spain \\ ${ }^{3}$ Institució Catalana de Recerca i Estudis Avançats (ICREA), Passeig Lluís Companys 23, ES-08010 Barcelona, Spain \\ ${ }^{4}$ Department of Applied Mathematics and Theoretical Physics (DAMTP), Centre for Mathematical Sciences, University of Cambridge, \\ Wilberforce Road, Cambridge CB3 OWA, United Kingdom
}

(Received 5 February 2020; accepted 16 April 2020; published 12 May 2020)

\begin{abstract}
Plasma balls are droplets of deconfined plasma surrounded by a confining vacuum. We present the first holographic simulation of their real-time evolution via the dynamics of localized, finite-energy black holes in the five-dimensional anti-de Sitter (AdS) soliton background. The dual gauge theory is four-dimensional $\mathcal{N}=4$ super Yang-Mills theory compactified on a circle with supersymmetry-breaking boundary conditions. We consider horizonless initial data sourced by a massless scalar field. Prompt scalar field collapse produces an excited black hole at the bottom of the geometry together with gravitational and scalar radiation. The radiation disperses to infinity in the noncompact directions and corresponds to particle production in the dual gauge theory. The black hole evolves toward the dual of an equilibrium plasma ball on a time scale longer than naively expected. This feature is a direct consequence of confinement and is caused by long-lived, periodic disturbances bouncing between the bottom of the AdS soliton and the AdS boundary.
\end{abstract}

DOI: 10.1103/PhysRevLett.124.191601

Introduction.-Holography [1-3] maps the quantum dynamics of hot, strongly coupled, non-Abelian plasmas to the classical dynamics of black hole horizons in asymptotically anti-de Sitter (AdS) spacetimes. This has provided interesting insights potentially relevant for the far-fromequilibrium properties of the quark-gluon plasma created in heavy ion collision (HIC) experiments (see, e.g., [4] for a review). The first examples [5-20] considered gravity models dual to conformal field theories (CFTs). Subsequent work extended the analysis to nonconformal theories [21-24] and to theories with phase transitions [25-31]. Despite this progress, all these studies fail to incorporate one essential feature of quantum chromodynamics (QCD): confinement. In particular, the gauge theories considered in those models lack a gapped and discrete spectrum of asymptotic states like the one expected in a confining theory.

The possibility of modeling a confining gauge theory with a gravity dual was initiated by [32], and the AdS soliton geometry [33] provides a concrete realization of such a gravity dual. This geometry consists of AdS space compactified on a circle that shrinks to zero size at a region

Published by the American Physical Society under the terms of the Creative Commons Attribution 4.0 International license. Further distribution of this work must maintain attribution to the author(s) and the published article's title, journal citation, and DOI. Funded by SCOAP . called the infrared (IR) bottom, smoothly capping off the spacetime there. The dual gauge theory is also compactified on a circle whose inverse size sets the confinement scale $\Lambda$. The AdS soliton is the preferred homogeneous phase at temperatures below the deconfinement temperature $T_{c}=\Lambda$. Above $T_{c}$, the preferred homogeneous phase is a translationally invariant black brane dual to an infinitely extended deconfined plasma. Homogeneous gravitational collapse in the AdS soliton that leads to black brane configurations was studied in [34].

It was conjectured, in [35], that localized, finite-energy black hole solutions also exist in the AdS soliton background and that they should be dual to stable, mixed-phase configurations in the gauge theory known as plasma balls: finite-size droplets of deconfined plasma surrounded by the confining vacuum. Subsequent work, see, for example, [36-43], confirmed this picture. In particular, [42] numerically constructed static solutions of finite-energy black holes localized at the IR bottom of the AdS soliton. It was found that small black holes of this type are well described by spherically symmetric Schwarzschild solutions that have $T \gg T_{c}$, whereas large black holes resemble "pancakes" with $T \gtrsim T_{c}$ and are well approximated by the AdS black brane solution at points away from the interface with the surrounding vacuum.

In a set of articles, we are initiating a new program to study the far-from-equilibrium physics of confining gauge 
theories with gravity duals. In these theories, any collision with sufficiently high but finite energy is expected to result in the formation of an excited plasma ball. Such collisions were analyzed in the approximation of linearized gravity in [44]. Although this captures the expected set of asymptotic states, the linear approximation is unable to describe the formation of a black hole horizon and its subsequent time evolution.

In this first Letter, we present the first fully nonlinear evolution of finite-energy black holes in a confining geometry. Specifically, we consider gravity in five spacetime dimensions with a negative cosmological constant coupled to a massless scalar field and solve for spacetimes with AdS soliton asymptotics. This gravity model is firmly embedded in string theory, since it is dual to a confining gauge theory [32] in four dimensions obtained by compactifying $\mathcal{N}=4, S U\left(N_{c}\right)$ super Yang-Mills (SYM) theory on a circle with supersymmetry-breaking boundary conditions.

Throughout the Letter, we relegate several technical details to the Supplemental Material [45].

Numerical Scheme.-The results presented in this Letter are obtained with a numerical code that solves the generalized harmonic form of the Einstein field equations. We obtain solutions $g_{\mu \nu}=\hat{g}_{\mu \nu}+h_{\mu \nu}$, where the deformation $h_{\mu \nu}$ is not small and the background metric $\hat{g}_{\mu \nu}$ is that of the AdS soliton

$\hat{g}=\frac{1}{\left(1-\rho^{2}\right)^{2}}\left(-d t^{2}+\frac{4 \rho^{2}}{f(\rho)} d \rho^{2}+d x_{1}^{2}+d x_{2}^{2}+f(\rho) d \theta^{2}\right)$,

with $f(\rho)=1-\left(1-\rho^{2}\right)^{4}$. We have set the AdS radius and the location of the AdS boundary to unity, so all coordinates are effectively dimensionless. However, we reinstate dimensions whenever we quote specific values below. In these coordinates, the IR bottom is at $\rho=0$ and the boundary directions $-\infty<t, x_{1}, x_{2}<\infty$ are noncompact. There are only three of these boundary directions, reflecting the fact that four-dimensional SYM theory compactified on a circle behaves effectively as a threedimensional theory at distances longer than the size of the circle.

In deforming the full metric away from the AdS soliton, we take the general form of the metric to be

$$
\begin{aligned}
g= & g_{t t} d t^{2}+g_{\rho \rho} d \rho^{2}+g_{x_{1} x_{1}} d x_{1}^{2}+g_{x_{2} x_{2}} d x_{2}^{2}+g_{\theta \theta} d \theta^{2} \\
& +2\left(g_{t \rho} d t d \rho+g_{t x_{1}} d t d x_{1}+g_{t x_{2}} d t d x_{2}\right. \\
& \left.+g_{\rho x_{1}} d \rho d x_{1}+g_{\rho x_{2}} d \rho d x_{2}+g_{x_{1} x_{2}} d x_{1} d x_{2}\right) .
\end{aligned}
$$

In other words, we assume no symmetries except for the $U(1)$ of the circle parametrized by $\theta$ which, furthermore, is assumed to be hypersurface orthogonal. The period $\Delta \theta$ of this circle sets both the confinement scale $\Lambda$ and the deconfinement temperature $T_{c}=1 / \Delta \theta=\Lambda$. Light rays along the $\rho$ direction take a boundary time $t_{\text {bounce }} \approx 0.8 \Lambda^{-1}$ to travel from the IR bottom to the AdS boundary and back. From the near-boundary falloff of the metric, we extract the gauge theory stress tensor which we multiply by a factor of $2 \pi^{2} / N_{c}^{2}$ so that the rescaled quantities are finite in the large$N_{c}$ limit. We choose a renormalization scheme in which the AdS soliton has a vanishing stress tensor which implies that a translationally invariant black brane has energy density and pressures

$\left(\epsilon, P_{1}=P_{2}, P_{\theta}\right)=\frac{\pi^{4}}{4}\left(3 T^{4}+\Lambda^{4}, T^{4}-\Lambda^{4}, T^{4}+3 \Lambda^{4}\right)$.

We couple gravity to a massless real scalar field $\varphi$. In the gauge theory, this means that we consider the coupled dynamics of the stress tensor and a scalar operator dual to $\varphi$. For concreteness in this Letter, we will only display the expectation values of the stress tensor. Since we do not turn on a source for this field, the equation of state of the dual gauge theory remains the same as in a CFT, namely $T_{\mu}^{\mu}=0$. We construct time-symmetric data on the initial-time slice by solving the Hamiltonian constraint subject to a freely chosen initial scalar field profile. We take the latter to be a superposition of Gaussian lumps of the form

$\varphi\left(\rho, x_{1}, x_{2}\right)=\left(1-\rho^{2}\right)^{4} A_{0} \exp \left[-R\left(\rho, x_{1}, x_{2}\right)^{2} / \delta^{2}\right]$,

where

$R\left(\rho, x_{1}, x_{2}\right)^{2}=\left(\rho-\rho_{c}\right)^{2}+\Lambda^{2}\left(x_{1}-x_{1 c}\right)^{2}+\Lambda^{2}\left(x_{2}-x_{2 c}\right)^{2}$,

and $\left(\rho_{c}, x_{1 c}, x_{2 c}\right)$ is the center of the lump.

Results.-We dynamically construct localized black holes via gravitational collapse of a superposition of lumps of the form (4). We have performed several simulations, including some that do not result in the formation of a black hole. For concreteness, in this Letter, we focus on a simulation with $A_{0}=1.0, \delta=0.2$, and varying $\rho_{c}, x_{1 c}, x_{2 c}$ for a superposition of three Gaussian lumps, but we have verified that the results that we describe here do not depend on the number of lumps, or the particular values of $A_{0}, \delta, \rho_{c}, x_{1 c}, x_{2 c}$. In order to introduce an initial anisotropy in the $x_{1}-x_{2}$ plane, we choose the three lumps to be centered at $\rho_{c}=x_{1 c}=0$ but at different locations along $x_{2}$ given by $\Lambda x_{2 c}=\{-0.1,0,0.1\}$. This results in the formation of a single black hole at the IR bottom whose horizon is initially more extended along $x_{2}$ than along $x_{1}$, with a total mass $M \approx 0.226 \Lambda$. The solution is invariant under $x_{1} \rightarrow-x_{1}$ and/or $x_{2} \rightarrow-x_{2}$, so figures will only show half of each axis.

Scalar and gravitational waves propagate outward in all spatial directions as the black hole relaxes to equilibrium. In this context, the timelike AdS boundary and the IR bottom play the role of a waveguide: as outgoing waves 


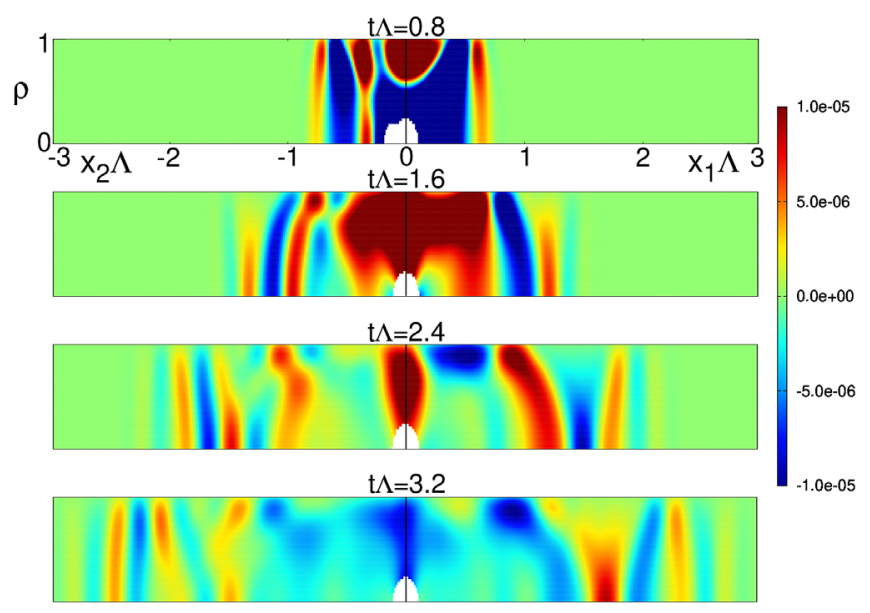

FIG. 1. Snapshots of the scalar field variable $\bar{\varphi}$ defined by $\varphi=\left(1-\rho^{2}\right)^{3} \bar{\varphi}$, at multiples of $t_{\text {bounce }} \approx 0.8 \Lambda^{-1}$. The scalar field $\varphi$ has the source turned off, and thus, in five spacetime dimensions, falls off as $\varphi \sim\left(1-\rho^{2}\right)^{4}$ near the boundary $\rho=1$. The excised region (shown in white) is a proper subset of the trapped region bounded by an apparent horizon that forms in the data. The left-hand (right-hand) half of each panel shows the data at $x_{1}=0$ for $x_{2}<0$ (at $x_{2}=0$ for $x_{1}>0$ ).

propagate along the boundary directions, these waves bounce back and forth between the boundary at $\rho=1$ and the IR bottom at $\rho=0$ at intervals given by $t_{\text {bounce. }}$ Figure 1 illustrates these dynamics with several snapshots of the scalar field variable.

In the vicinity of the black hole in the $x_{1}-x_{2}$ plane, there is a long-wavelength feature extending in the $\rho$ direction that is left behind by waves without enough momentum to escape in the boundary directions, but whose wavelengths are too long to be efficiently absorbed by the black hole. The outgoing wavefronts are evident as the snapshots progress in time, propagating in the boundary directions. The initial anisotropy in the $x_{1}-x_{2}$ plane is imprinted in the outgoing waves as differences in the spatial profiles along the $x_{1}$ and $x_{2}$ directions, as well as in the shape of the apparent horizon.

As outgoing waves disperse away into the asymptotic region $x_{1}, x_{2} \rightarrow \pm \infty$, the scalar field is expected to be described by a superposition of the discrete and gapped set of normal modes of the pure AdS soliton background. Each mode is characterized by a radial quantum number $n$ with a corresponding radial profile $\Psi_{n}(\rho)$, a momentum $k_{a}$ in the $x^{a}$ direction, and an energy $\omega_{n}(k)^{2}=k^{2}+m_{n}^{2}$. In the gauge theory, these are precisely the asymptotic states of masses $m_{n}$ of the confining vacuum. The projections into these modes are

$$
\phi_{n, \vec{k}}(t) \equiv \int d^{2} x_{a} \int d \rho \mu(\rho) \varphi\left(t, \rho, x_{a}\right) e^{i \vec{k} \cdot \vec{x}} \Psi_{n}(\rho)
$$

where $\mu(\rho)=2 \rho /\left(1-\rho^{2}\right)$ is the measure factor that ensures unit normalization of the $\Psi_{n}$. At asymptotically

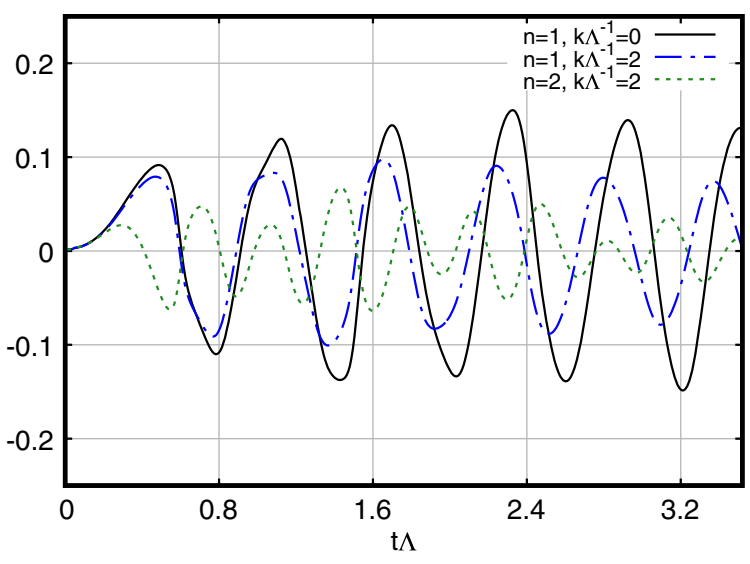

FIG. 2. Projections $\phi_{n, \vec{k}}(t)$ of the bulk scalar field $\varphi$ onto the normal modes of the AdS soliton according to (6), with $k_{1}=k_{2} \equiv k$. Times indicated on the horizontal axis are in multiples of $t_{\text {bounce }} \approx 0.8 \Lambda^{-1}$.

late times, we expect the different modes to decouple from one another and, hence, that $\phi_{n, \vec{k}}(t) \rightarrow c_{n, \vec{k}} \exp \left[-i \omega_{n}(k) t\right]$, where the coefficients $c_{n, \vec{k}}$ are time independent and each mode oscillates with well-defined frequency $\omega_{n}(k)$. Figure 2 shows the result of applying (6) to project the scalar field $\varphi\left(t, \rho, x_{a}\right)$ from the fully nonlinear evolution into some of the lowest-lying modes. Table I lists the frequencies $\omega_{n}(k)$ extracted from sinusoidal fits to these projections within different time windows. On the one hand, we see that these frequencies approach the known normal frequencies of a scalar field in the AdS soliton [46] as time progresses, showing that the late-time behavior can be approximately interpreted in terms of the expected asymptotic states. On the other hand, the small discrepancies in the frequencies at the latest time window, and the fact that the projections shown in Fig. 2 still exhibit amplitude modulations, suggest that nonlinear mode mixing is still taking place for the evolution times considered here. In gauge theory language, complete freeze-out has not yet been reached.

It is illustrative to see the time evolution from the viewpoint of the boundary stress tensor. Figure 3 shows a snapshot of the energy density, whose bulk dual is the finite-energy black hole depicted in the second panel of Fig. 1. Dispersing waves in the bulk give rise to the ripples visible in Fig. 3, leaving behind a black hole that is imprinted on the boundary as a central lump of localized energy density that oscillates around a nonzero spatial profile as it settles down to equilibrium. We have checked that the late-time energy-density profile of this dynamical plasma ball is approaching the profile of an equilibrium plasma ball of [42] with a temperature $T_{\text {equil }} \approx 2.1 T_{c}$ (see below). This means that, at late times, our plasma balls are better approximated by small and nearly spherically symmetric Schwarzschild black holes than by pancakelike configurations. The spacelike eigenvalues $P_{1}, P_{2}, P_{\theta}$ of 
TABLE I. Frequencies $\omega_{n}(k) / \Lambda$ of some low-lying modes with $k_{1}=k_{2}=k$ extracted from sinusoidal fits to the projections $\phi_{n, \vec{k}}(t)$ shown in Fig. 2. The first three entries in each cell correspond to fits within the three time windows $t \Lambda \in(0,1.6),(0.8,2.4),(1.6,3.2)$, respectively. The values inside the parentheses correspond to the normal frequencies of the AdS soliton.

\begin{tabular}{lcccc}
\hline \hline & $k / \Lambda=0$ & $k / \Lambda=2$ & $k / \Lambda=4$ & $k / \Lambda=6$ \\
\hline$n=1$ & $9.6,10.4,10.5(10.7)$ & $9.8,10.7,10.9(11.1)$ & $10.9,11.7,11.8(12.1)$ & $11.7,13.1,13.6(13.7)$ \\
$n=2$ & $16.8,17.6,18.4(18.5)$ & $17.0,17.8,18.6(18.7)$ & $17.5,18.2,19.0(19.3)$ & $19.8,20.0,20.2(20.3)$ \\
\hline \hline
\end{tabular}

the boundary stress tensor correspond to the pressures in the local rest frame. Figure 4 depicts the time dependence of all the eigenvalues of the stress tensor at the center of the plasma ball, $x_{1}=x_{2}=0$. Each curve in Fig. 4 is periodically disturbed, first at $t=t_{\text {bounce }} / 2$ when waves from the IR bottom first reach the boundary and, thereafter, in intervals of $\Delta t=t_{\text {bounce. }}$ To emphasize the fact that these bounces are associated with the AdS soliton geometry in Fig. 4, we also show the energy density for a simulation that does not lead to the formation of a black hole.

Spatial profiles of the gauge theory energy density are shown in Fig. 5 at several representative times. As waves from the IR bottom reach the boundary, which happens around $t=t_{\text {bounce }} / 2$, the shorter-wavelength spatial inhomogeneities in the IR become more evidently imprinted in the boundary one-point functions. These short-wavelength features are subsequently either (i) carried away to the asymptotic region $x_{1}, x_{2} \rightarrow \pm \infty$ by outgoing waves with momentum in the boundary directions, or (ii) carried by radially propagating waves back to the vicinity of the black hole, where they are efficiently absorbed. Both mechanisms for shedding short-wavelength features are consistent with the bulk snapshots in Fig. 1. For comparison, Fig. 5 also shows the spatial profile of an equilibrium plasma ball with $T=2.1 T_{c}$ [42]. We see that this is close to, but not exactly on top of, the profile of the latest-time dynamical plasma ball. This small discrepancy is expected because the dynamical ball has not yet equilibrated at the time shown. Moreover, the discrepancy is larger away from the center of

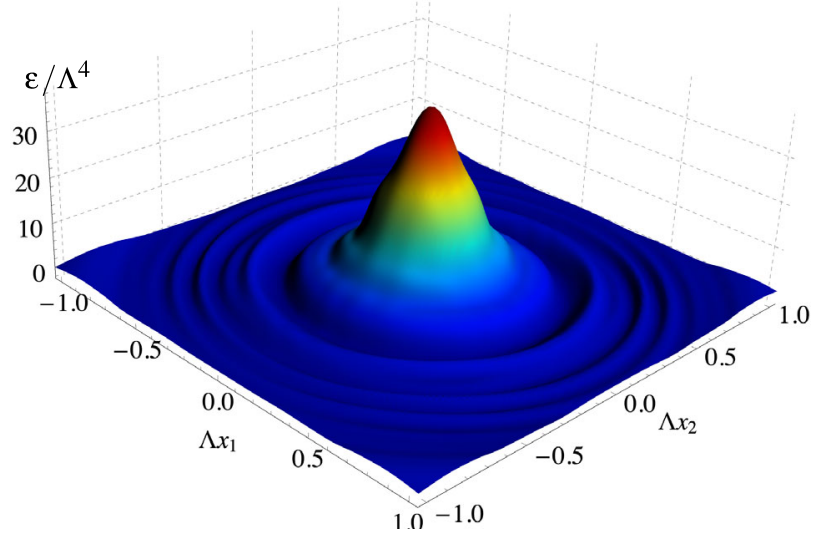

FIG. 3. Snapshot of the gauge theory energy density at $t=1.6 \Lambda^{-1} \approx 2 t_{\text {bounce }}$. the ball, since there, the oscillations are more pronounced than at the center.

Discussion.-We have presented the first study of the real-time evolution for finite-energy black holes in the AdS soliton background. The black holes are dual to plasma balls: localized droplets of deconfined matter surrounded by the confining vacuum. These objects are interesting because a collision with sufficiently high but finite energy in a confining theory with a gravity dual will generically produce an excited plasma ball.

Our results suggest that small excited plasma balls relax to classically nonlinearly stable configurations with longlived excitations. Qualitatively, the radiation emitted during the relaxation process can be understood as consisting of two components. The first one disperses away to infinity in the noncompact directions, and at late times, it has an interpretation in terms of individual particles. The entropy carried away by these modes is $O(1)$ and, hence,

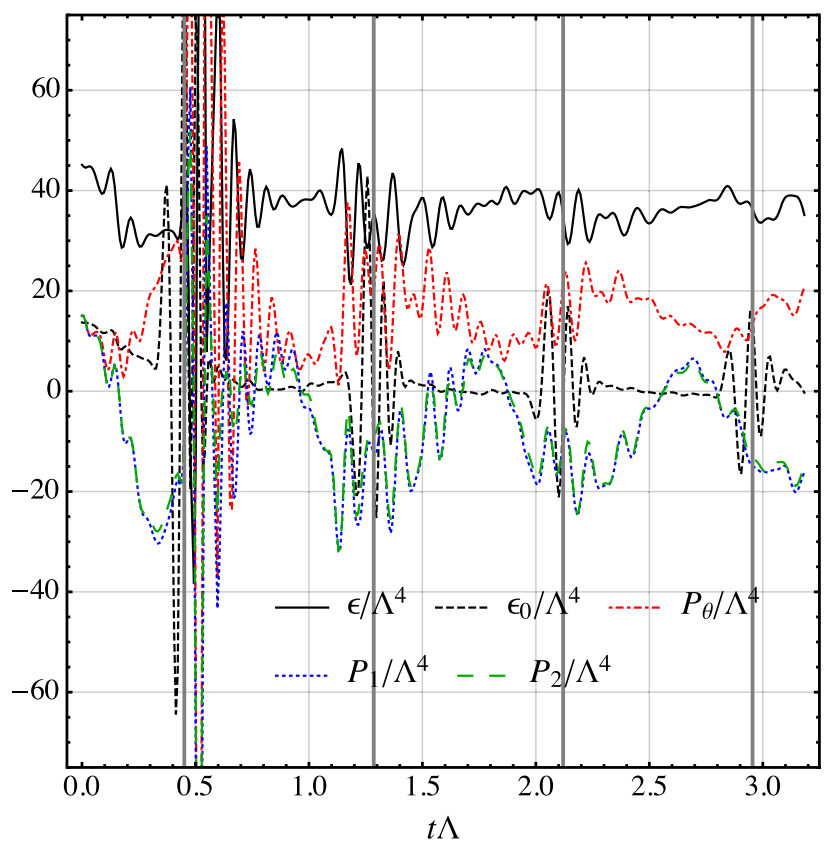

FIG. 4. Gauge theory energy density and pressures at the center of the plasma ball, $x_{1}=x_{2}=0$. At this point, there is no difference between the lab frame and the local rest frame. The energy density $\epsilon_{0}$ for a simulation that does not produce a black hole is also shown. Peaks occur in intervals of $t_{\text {bounce }} \approx 0.8 \Lambda^{-1}$, emphasized by the vertical lines that begin at the first peak and are separated by $t_{\text {bounce. }}$ 


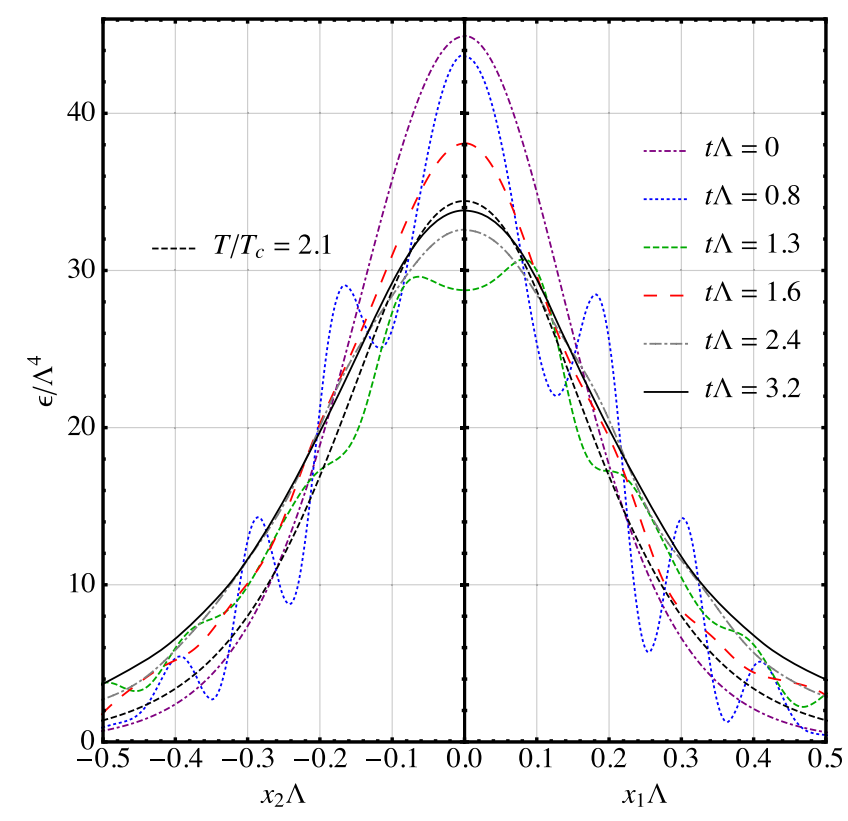

FIG. 5. Gauge theory energy density at several representative times. The left (right) half of the plot shows the data at $x_{1}=0$ $\left(x_{2}=0\right)$. The $T / T_{c}=2.1$ curve corresponds to an equilibrium plasma ball [42].

subleading with respect to the $O\left(N_{c}^{2}\right)$ entropy that remains in the plasma ball. In other words, in our classical approximation, the radiated field is a coherent field with effectively zero entropy. In this approximation, the black hole and the dual plasma ball eventually settle down to equilibrium, whereas in a finite- $N_{c}$ theory like QCD, the black hole would Hawking evaporate and the plasma ball would hadronize. The second component of the radiation is associated with long-lived waves bouncing back and forth between the IR bottom and the AdS boundary at intervals $t_{\text {bounce }} \approx 0.8 \Lambda^{-1}$. These waves cause periodic disturbances of the black hole and lead to an equilibration time longer than what would be naively expected. For example, an estimate based on the final temperature would give $\Lambda t_{\text {equil }} \approx$ $\Lambda / T_{\text {equil }} \approx 0.5$ for the plasma ball studied here.

Our analysis suggests that these long-lived disturbances are a robust feature of small, finite-energy black holes in a confining background, or equivalently, of small plasma balls in large- $N_{c}$ gauge theories with a gravity dual. This feature can be understood as follows. Modes with wavelengths much longer than the size of the black hole interact weakly with it and easily disperse away in the noncompact directions, and modes with wavelengths much shorter than the size of the black hole are efficiently absorbed by it. Meanwhile, modes with wavelengths comparable to the size of the black hole interact strongly enough to be attracted to it and, hence, do not disperse away easily, but at the same time, are not efficiently absorbed by it. Therefore, these waves can bounce back and forth between the IR bottom and the AdS boundary.
In the gauge theory, these periodic disturbances correspond to a periodic transfer of energy between IR and ultraviolet (UV) modes inside the plasma ball, coupled to oscillations in the shape of the ball. We have seen that they have an important effect on the dynamics of the plasma ball that we have considered. Since its mass is $M<\Lambda$, this plasma ball is not a good model for the fireball created in a HIC. For this reason, we are currently investigating whether or not the periodic disturbances persist for large plasma balls. The obvious next step in our program is the study of finite-energy black hole collisions, which is a natural mechanism by which these large plasma balls can be formed. These will offer new holographic insights on confinement and finite-size effects in the equilibration of large- $N_{c}$, strongly coupled gauge theories. Some aspects of these theories will certainly differ from those in finite- $N_{c}$ theories. For example, the final state in these theories at asymptotically late times will contain an equilibrated plasma ball that will not hadronize. However, some features in the evolution may be similar between finite and infinite $N_{c}$. Assessing which ones will require further analyses beyond this first study.

We thank Jay Armas, Yago Bea, Alex Buchel, Jorge Casalderrey-Solana, Paul Romatschke, and Christopher Rosen for valuable discussions and comments. H. B. and P. F. are supported by the European Research Council Grant No. ERC-2014-StG 639022-NewNGR. P. F. is also supported by a Royal Society University Research Fellowship (Grant No. UF140319). D. M. is supported by the Spanish Government under Grants No. FPA2016-76005-C2-1-P, No. FPA2016-76005-C2-2-P, No. SGR-2017-754, and No. MDM-2014-0369. Simulations were run on the Perseus cluster at Princeton University and the MareNostrum 4 (Grant No. FI-2019-1-0010) cluster at the Barcelona Supercomputing Center.

*h.bantilan@qmul.ac.uk p.figueras@qmul.ac.uk \#mateos@fqa.ub.edu

[1] J. M. Maldacena, Adv. Theor. Math. Phys. 2, 231 (1998).

[2] S. Gubser, I. R. Klebanov, and A. M. Polyakov, Phys. Lett. B 428, 105 (1998).

[3] E. Witten, Adv. Theor. Math. Phys. 2, 253 (1998).

[4] J. Casalderrey-Solana, H. Liu, D. Mateos, K. Rajagopal, and U.A. Wiedemann, Gauge/String Duality, Hot QCD and Heavy Ion Collisions (Cambridge University Press, Cambridge, England, 2011).

[5] P. M. Chesler and L. G. Yaffe, Phys. Rev. Lett. 102, 211601 (2009).

[6] P. M. Chesler and L. G. Yaffe, Phys. Rev. D 82, 026006 (2010).

[7] P. M. Chesler and L. G. Yaffe, Phys. Rev. Lett. 106, 021601 (2011).

[8] M. P. Heller, R. A. Janik, and P. Witaszczyk, Phys. Rev. Lett. 108, 201602 (2012). 
[9] H. Bantilan, F. Pretorius, and S. S. Gubser, Phys. Rev. D 85, 084038 (2012).

[10] M. P. Heller, D. Mateos, W. van der Schee, and D. Trancanelli, Phys. Rev. Lett. 108, 191601 (2012).

[11] M. P. Heller, R. A. Janik, and P. Witaszczyk, Phys. Rev. D 85, 126002 (2012).

[12] M. P. Heller, D. Mateos, W. van der Schee, and M. Triana, J. High Energy Phys. 09 (2013) 026.

[13] J. Casalderrey-Solana, M. P. Heller, D. Mateos, and W. van der Schee, Phys. Rev. Lett. 111, 181601 (2013).

[14] J. Casalderrey-Solana, M. P. Heller, D. Mateos, and W. van der Schee, Phys. Rev. Lett. 112, 221602 (2014).

[15] H. Bantilan and P. Romatschke, Phys. Rev. Lett. 114, 081601 (2015).

[16] P. M. Chesler and L. G. Yaffe, J. High Energy Phys. 10 (2015) 070.

[17] P. M. Chesler, Phys. Rev. Lett. 115, 241602 (2015).

[18] P. M. Chesler and W. van der Schee, Int. J. Mod. Phys. E 24, 1530011 (2015).

[19] P. M. Chesler, J. High Energy Phys. 03 (2016) 146.

[20] J. Casalderrey-Solana, D. Mateos, W. van der Schee, and M. Triana, J. High Energy Phys. 09 (2016) 108.

[21] A. Buchel, M. P. Heller, and R. C. Myers, Phys. Rev. Lett. 114, 251601 (2015).

[22] M. Attems, J. Casalderrey-Solana, D. Mateos, I. Papadimitriou, D. Santos-Olivan, C. F. Sopuerta, M. Triana, and M. Zilhao, J. High Energy Phys. 10 (2016) 155.

[23] M. Attems, J. Casalderrey-Solana, D. Mateos, D. Santos-Olivan, C. F. Sopuerta, M. Triana, and M. Zilhao, J. High Energy Phys. 01 (2017) 026.

[24] M. Attems, J. Casalderrey-Solana, D. Mateos, D. Santos-Olivan, C. F. Sopuerta, M. Triana, and M. Zilhao, J. High Energy Phys. 06 (2017) 154.

[25] R. A. Janik, J. Jankowski, and H. Soltanpanahi, Phys. Rev. Lett. 117, 091603 (2016).

[26] R. A. Janik, J. Jankowski, and H. Soltanpanahi, J. High Energy Phys. 06 (2016) 047.

[27] M. Attems, Y. Bea, J. Casalderrey-Solana, D. Mateos, M. Triana, and M. Zilhao, J. High Energy Phys. 06 (2017) 129.

[28] R. A. Janik, J. Jankowski, and H. Soltanpanahi, Phys. Rev. Lett. 119, 261601 (2017).
[29] M. Attems, Y. Bea, J. Casalderrey-Solana, D. Mateos, M. Triana, and M. Zilhao, Phys. Rev. Lett. 121, 261601 (2018).

[30] M. Attems, Y. Bea, J. Casalderrey-Solana, D. Mateos, and M. Zilhao, J. High Energy Phys. 01 (2020) 106.

[31] L. Bellantuono, R. A. Janik, J. Jankowski, and H. Soltanpanahi, J. High Energy Phys. 10 (2019) 146.

[32] E. Witten, Adv. Theor. Math. Phys. 2, 505 (1998).

[33] G. T. Horowitz and R. C. Myers, Phys. Rev. D 59, 026005 (1998).

[34] B. Craps, E. J. Lindgren, and A. Taliotis, J. High Energy Phys. 12 (2015) 116.

[35] O. Aharony, S. Minwalla, and T. Wiseman, Classical Quantum Gravity 23, 2171 (2006).

[36] S. Lahiri and S. Minwalla, J. High Energy Phys. 05 (2008) 001 .

[37] S. Bhardwaj and J. Bhattacharya, J. High Energy Phys. 03 (2009) 101.

[38] M. M. Caldarelli, O. J. C. Dias, R. Emparan, and D. Klemm, J. High Energy Phys. 04 (2009) 024.

[39] V. Cardoso and O. J. C. Dias, J. High Energy Phys. 04 (2009) 125.

[40] R. Emparan and G. Milanesi, J. High Energy Phys. 08 (2009) 012.

[41] V. Cardoso, O. J. C. Dias, and J. V. Rocha, J. High Energy Phys. 01 (2010) 021.

[42] P. Figueras and S. Tunyasuvunakool, J. High Energy Phys. 06 (2014) 025.

[43] J. Armas, J. Bhattacharya, and N. Kundu, J. High Energy Phys. 06 (2016) 015.

[44] V. Cardoso, R. Emparan, D. Mateos, P. Pani, and J. V. Rocha, J. High Energy Phys. 01 (2014) 138.

[45] See Supplemental Material at http://link.aps.org/ supplemental/10.1103/PhysRevLett.124.191601 for definitions of all evolved variables, details of how the gauge choice is implemented, all boundary conditions, and all parameters related to excision and time evolution. It includes a discussion of how the boundary stress tensor is obtained from the bulk data, and numerical tests demonstrating convergence to a solution of the Einstein field equations.

[46] C. Csaki, H. Ooguri, Y. Oz, and J. Terning, J. High Energy Phys. 01 (1999) 017. 